\title{
LOS PLANES DE ACCIÓN COMERCIAL DE LA COMU- NIDAD VALENCIANA. EVALUACIÓN DE LOS CASOS DE ALICANTE Y ELCHE
}

\author{
Ana ESPINOSA SEGUÍ \\ Universidad de Alicante \\ Departamento de Geografía Humana \\ Email: ana.espinosa@ua.es
}

\author{
Maria D. DE JUAN \\ Universidad de Alicante \\ Departamento de Marketing \\ Email: mayo@ua.es
}

\author{
Recibido: 04/10/2013
}

Aceptado: 13/02/2014

RESUMEN: Con la intención de aunar los estudios comerciales con los urbanísticos en las ciudades valencianas, el Consejo Superior de Cámaras de Comercio de la Comunidad Valenciana desarrolló los Planes de Acción Comercial (PAC) en las principales ciudades de la provincia de Alicante. Estos documentos consultivos, realizados para impulsar la investigación urbana y comercial a escala local, han pretendido orientar, proponer y financiar actuaciones de intervención a las administraciones locales. En este artículo, se revisa la trayectoria de los PAC y se analizan, en especial, los resultados de las acciones llevadas a cabo en las ciudades de Alicante y Elche, con el objetivo de conocer el estado de situación de estas propuestas urbano- comerciales.

PALABRAS CLAVE: Plan de Acción Comercial, Comunidad Valenciana, evaluación de resultados, urbanismo comercial, comercio urbano.

THE "PLANES DE ACCIÓN COMERCIAL" OF THE COMMUNITY OF VALENCIA. EVALUATION OF THE ALICANTE AND ELCHE CASES

ABSTRACT: In order to homogenize the urban studies in the Valencian cities and towns, UrbanCommercial Action Plans (CAP) have been promoted by the regional government of Valencia (Spain). These consultative documents, made to promote urban and commercial research at a local

1 El presente texto se incardina en el proyecto de investigación Las ciudades españolas en la etapa autonómica (1978-2012). Dinámicas, procesos y políticas (URBSPAIN), financiado por el Plan Nacional de Investigación I+D+i del Ministerio de Economía y Competitividad para el período 2010-2013 (Código CSO 2009-11261-Subpr. GEOG). 
scale basis, have sought to guide, propose and finance actions concerning the local government intervention. We present a revision of the trajectory of the PAC and analyse, in particular, the results of the activities carried out in the cities of Alicante and Elche with the objective of update the state of the art about these commercial urban proposals.

KEY WORDS: Commercial Action Plan, Region of Valencia, performance assessment, urban commerce, urban retail.

\section{INTRODUCCIÓN}

El comercio es una de las actividades económicas más representativas de los espacios urbanos, ya que aporta vitalidad y funcionalidad a la ciudad, proporciona empleo a los comerciantes y abastece a los ciudadanos (AldeiturRiagA, 2004).

Aunque es indudable que la presencia de un comercio local saludable contribuye a mantener una ciudad viva y funcional, e incluso se le puede atribuir una gran capacidad regeneradora en áreas urbanas en crisis, no ha sido hasta la década de los años noventa, cuando la geografía comercial y la administración pública han decidido unificar los estudios comerciales a los proyectos urbanos de mayor envergadura a escala local e incluso regional.

De esta intensificación de las relaciones urbano-comerciales ha surgido una nueva estrategia de intervención en la ciudad conocida como Urbanismo Comercial, en la que el comercio local es visto como un pilar básico en los procesos de intervención urbana y la mayoría de las acciones emprendidas en la ciudad se hacen por y para el comercio (Pérez, 1998; Aparicio de Castro, Tejada BarrenetXea, Zorrilla Calvo, 2010).

Incluso en espacios urbanos de muy reducidas dimensiones, el enfoque de intervención en el espacio con una perspectiva comercial ha dado como resultado proyectos de microurbanismo comercial, en los que aspectos tales como el mobiliario urbano, la iluminación, la peatonalización o la ordenación del tráfico rodado tienen una lectura directa en la oferta y demanda comercial local (véase, por ejemplo, la actuación de microurbanismo propuesta para Ciutat Vella en Barcelona, en 2010 centrada en zonas muy concretas como el Macba/CCCB) e incluso turística (Getz, 1993; Perles Ribes, 2011 ).

En España, una de las comunidades autónomas que primero adoptó el Urbanismo Comercial como medio para recuperar espacios urbanos en crisis fue la Comunidad Valenciana, a través de los Planes de Acción Comercial que lleva realizando el Consejo de Cámaras de Comercio, Industria y Navegación de la 
Comunidad Valenciana, a través de la Oficina PATECO, desde el año 1998 (véase TARRAGÓ, 2012 para una interesante revisión sobre el urbanismo comercial para Europa en general y España en particular).

Los Planes de Acción Comercial, en adelante PAC, son instrumentos consultivos, orientativos y no vinculantes utilizados a escala local o supramunicipal y encargados de definir un programa de actuaciones que permita dinamizar la actividad comercial y de servicios del ámbito territorial donde se desarrollan (www.pateco.org).

La promoción de los PAC es local, con el apoyo e impulso de la Generalitat Valenciana, mediante ayudas públicas para la realización del propio estudio y la ejecución de las obras de mejora urbano-comercial que se consideren oportunas.

En la realización de los Planes se implica activamente a los empresarios de la ciudad. La idea es generar un marco de trabajo, diálogo, consenso y compromiso entre el Ayuntamiento, las asociaciones de comerciantes y los colectivos ciudadanos en un objetivo común, que es conseguir la revitalización de la ciudad.

Los empresarios individuales y los consumidores participan en la elaboración del PAC a través de dinámicas y reuniones de grupo, en las que pueden expresar libremente su opinión sobre la situación del comercio local. Otros agentes económicos locales y administraciones participan a través de la comisión mixta, que se reúne periódicamente a iniciativa del Ayuntamiento.

Hasta 2013, se habían desarrollado 71 PAC en toda la Comunidad Valenciana, afectando a 271 ciudades y pueblos, que concentran el $72 \%$ de la población regional. Esta amplia cobertura territorial de los PAC ha sido apoyada desde la Conselleria de Economía, Industria y Turismo, y sobre todo, desde la nueva Ley 3/2011, de 23 de marzo, de Comercio de la Comunidad Valenciana. Así, en el artículo 39 "Planes de acción comercial y planes directores de comercio", la Generalitat Valenciana se compromete a impulsar la realización de aquellos PAC municipales y supramunicipales que los ayuntamientos desarrollen con el fin de obtener una serie de objetivos y propuestas de actuación para los municipios a corto, medio y largo plazo. Para la consecución de los PAC se facilita el diálogo con los agentes sociales, económicos y comerciales del municipio, que deben participar en la fase de diagnóstico y de propuestas de mejora. Y una vez finalizados los estudios, los gobiernos locales o entes territoriales competentes pueden solicitar a la Generalitat Valenciana una línea de ayudas anual o bianual para financiar parcialmente las acciones priorizadas desde la escala local, atendiendo a las necesidades más urgentes. 
Los PAC han utilizado una misma metodología en todos los municipios donde se han desarrollado, en la que se incluía un diagnóstico pormenorizado del municipio, la delimitación de los principales ejes y zonas comerciales urbanas y un plan de actuación con propuestas de urbanismo y macrourbanismo comercial para el municipio.

A pesar de su orientación claramente comercial, los PAC se han convertido en documentos de consulta de información municipal de temática económica, comercial, social y demográfica, ya que suponen una radiografía actualizada del municipio o conjunto de municipios que analizan, sobre todo en la fase del diagnóstico.

La sección de propuestas de los PAC, sin embargo, es en realidad un catálogo de buenas prácticas urbano-comerciales orientado a cada municipio. Estas sugerencias que se ofrecían desde el documento, estaban orientadas a distintos temas vitales en el buen funcionamiento del comercio, desde líneas de modernización y mejora de la gestión comercial de los establecimientos comerciales, hasta programas de dinamización turística y comercial, sin olvidar por supuesto, el urbanismo comercial, verdadero motor impulsor de los PAC.

Por su condición de instrumentos consultivos, orientativos y no vinculantes, las propuestas que proponen los PAC han tenido una ejecución desigual en muchos municipios, limitándose en ocasiones a cumplir algunas recomendaciones puntuales en acciones de poca envergadura que sugerían los técnicos de la Oficina PATECO.

Es decir, que a pesar de disponer de un estudio minucioso de las necesidades de los municipios estudiados, de las acciones concretas para llevar a cabo, de una financiación estable y accesible desde el gobierno regional, en demasiadas ocasiones los PAC se han quedado como documentos de consulta y no ejecutivos.

Por ese motivo, en este artículo se presentan los resultados de una valoración realizada en los municipios que cuentan con un PAC en la provincia de Alicante, centrando el estudio sobre todo en las ciudades de Alicante y Elche. El objetivo principial es evaluar el impacto e influencia de los Planes de Acción Comercial en la ejecución de acciones urbano-comerciales llevadas a cabo a escala local y detectar el grado de satisfacción de los resultados obtenidos tras la puesta en marcha de las propuestas. 


\section{METODOLOGÍA}

El presente análisis de los Planes de Acción Comercial de la Comunidad Valenciana se ha centrado en evaluar el papel de estas herramientas de mejora urbano-comercial en el territorio. Dado que las propuestas que proponen los PAC no son de obligado cumplimiento por parte de los ayuntamientos o entes territoriales competentes, se ha querido estudiar la materialización de las acciones que han propuesto los PAC, la priorización de las propuestas y el diseño final que los ayuntamientos han dado a sus ciudades tomando en cuenta las consideraciones expuestas en sus respectivos Planes de Acción Comercial.

El ámbito de estudio ha sido delimitado a los municipios alicantinos que contaban con un PAC en el año 2013, realizando un análisis mucho más detallado en las dos principales ciudades de la provincia, Alicante y Elche, motores comerciales de gran parte del territorio provincial.

De un universo de 25 municipios, en este estudio han participado 10, lo que significa una cobertura del 40 por ciento.

En esta investigación se ha considerado necesario comparar municipios con estructuras sociales, económicas y comerciales diversas para evaluar el impacto de las propuestas sobre territorios con diferente idiosincrasia.

A pesar de que los PAC han desarrollado un método de trabajo similar que se ha aplicado a todos los estudios realizados, las propuestas emitidas en cada uno de los proyectos han estado influidas por las características particulares de cada municipio. A este respecto se han tenido en cuenta variables como el tamaño, la base económica, la localización, la población vinculada al municipio y la estructura comercial local. En relación a esta última variable se ha considerado el grado de suburbanización comercial, el volumen de comercio local independiente y la localización de los comercios, entre otras variables.

Con el fin de poder comparar todo el repertorio de propuestas de los PAC, independientemente de las excepcionalidades que presenta cada municipio, todas las acciones detectadas en los municipios objeto de estudio (ejecutadas o no) se han agrupado en cinco grandes grupos: urbanismo comercial (UC), mejora de la gestión comercial (GC), dinamización comercial (DC), dinamización turísticocomercial (DTC) y modernización de establecimientos comerciales (MEC) (Vid. Cuadros 1 al 5). 


\begin{tabular}{|c|c|c|}
\hline \multicolumn{3}{|c|}{ Cuadro 1. Propuestas de urbanismo comercial (UC) } \\
\hline Relacionada con: & $\begin{array}{l}\text { Código de } \\
\text { propuesta }\end{array}$ & Línea de actuación \\
\hline \multirow{3}{*}{ Aparcamientos } & UC-Ap.1 & Apertura de nuevos aparcamientos públicos \\
\hline & UC-Ap. 2 & $\begin{array}{l}\text { Colocación de bolardos o pilonas para evitar } \\
\text { aparcamientos indebidos y para proteger a los } \\
\text { peatones }\end{array}$ \\
\hline & UC-Ap. 3 & $\begin{array}{l}\text { Implantación del sistema ORA de aparcamiento } \\
\text { en nuevas calles comerciales }\end{array}$ \\
\hline \multirow{2}{*}{ Mobiliario urbano } & UC-Mob. 1 & $\begin{array}{l}\text { Dotación de mobiliario urbano para la decora- } \\
\text { ción y la señalización de edificios y zonas peato- } \\
\text { nales }\end{array}$ \\
\hline & UC-Mob. 2 & $\begin{array}{l}\text { Dotación de mobiliario urbano para la ilumina- } \\
\text { ción }\end{array}$ \\
\hline \multirow{3}{*}{$\begin{array}{l}\text { Espacio } \\
\text { público }\end{array}$} & UC-Epúb. 1 & $\begin{array}{l}\text { Optimización de la ocupación de espacio públi- } \\
\text { co por terrazas de restaurantes }\end{array}$ \\
\hline & UC-Epúb. 2 & Peatonalización de calles comerciales \\
\hline & UC-Epúb. 3 & $\begin{array}{l}\text { Conversiones de calles con mucho tráfico en ca- } \\
\text { lles de coexistencia. Por ej. ampliación de aceras } \\
\text { y reducción de carriles de tráfico rodado }\end{array}$ \\
\hline
\end{tabular}

\begin{tabular}{|l|l|l|}
\hline \multicolumn{2}{|l|}{ Cuadro 2. Propuestas de mejora de la gestión comercial $(G C)$} \\
\hline Relacionada con: & $\begin{array}{l}\text { Código de } \\
\text { propuesta }\end{array}$ & Línea de actuación \\
\hline Formación & GC-Form1 & $\begin{array}{l}\text { Cursos de formación comercial sugeridos por el } \\
\text { PAC y financiados (total o parcialmente) por la } \\
\text { Generalitat Valenciana }\end{array}$ \\
\hline Asociacionismo & GC-Aso1 & $\begin{array}{l}\text { Fomento del asociacionismo comercial a escala } \\
\text { local }\end{array}$ \\
\hline FUENTE: Elaboración propia
\end{tabular}

\begin{tabular}{|l|l|l|}
\hline \multicolumn{2}{|l|}{ Cuadro 3. Propuestas de dinamización comercial (DC) } \\
\hline Relacionada con: & $\begin{array}{l}\text { Código de } \\
\text { propuesta }\end{array}$ & Línea de actuación \\
\hline \multirow{2}{*}{ Imagen de la zona } & DC-Img.1 & $\begin{array}{l}\text { Creación de una imagen de marca comercial } \\
\text { local }\end{array}$ \\
\cline { 2 - 3 } & DC-Img. 2 & Creación de rutas comerciales peatonales \\
\hline
\end{tabular}




\begin{tabular}{|l|l|l|}
\hline \multirow{2}{*}{ Comunicación } & DC-Com.1 & $\begin{array}{l}\text { Campañas de promoción comercial puntuales } \\
\text { (día de la madre, del padre, rebajas, etc.) }\end{array}$ \\
\cline { 2 - 3 } & DC-Com.2 & $\begin{array}{l}\text { Realización de eventos de venta en la calle como } \\
\text { ferias o ferias } \text { outlet. }\end{array}$ \\
\cline { 2 - 3 } & DC-Com.3 & $\begin{array}{l}\text { Creación de guías comerciales (en la página web } \\
\text { local o en folletos) }\end{array}$ \\
\hline
\end{tabular}

\begin{tabular}{|l|l|l|}
\hline \multicolumn{2}{|l|}{ Cuadro 4. Propuestas de dinamización turístico comercial (DTC) } \\
\hline Relacionada con: & $\begin{array}{l}\text { Código de } \\
\text { propuesta }\end{array}$ & Línea de actuación \\
\hline Imagen de la zona & DTC-Img.1 & Creación de rutas turístico-comerciales \\
\hline Comunicación & $\begin{array}{l}\text { DTC- } \\
\text { Com.1 }\end{array}$ & $\begin{array}{l}\text { Animación y promoción turístico-comercial con- } \\
\text { junta con hoteles y restaurantes }\end{array}$ \\
\hline \multicolumn{2}{|l}{ FuENTE: Elaboración propia } \\
\hline
\end{tabular}

\begin{tabular}{|l|l|l|}
\hline \multicolumn{2}{|c|}{ Cuadro 5. Propuestas de modernización de establecimientos comerciales (MEC) } \\
\hline Relacionada con: & $\begin{array}{l}\text { Código de } \\
\text { propuesta }\end{array}$ & Línea de actuación \\
\hline Tecnología & MEC-Tec.1 & $\begin{array}{l}\text { Sugerencias para modernizar el equipo informá- } \\
\text { tico de los comercios (con y sin subvención del } \\
\text { gobierno regional) }\end{array}$ \\
\hline $\begin{array}{l}\text { Infraestructuras } \\
\text { de los comercios }\end{array}$ & MEC-Inf.1 & $\begin{array}{l}\text { Propuestas para reformas de locales comerciales } \\
\text { (con subvención del gobierno regional) }\end{array}$ \\
\hline $\begin{array}{l}\text { Innovación mino- } \\
\text { rista }\end{array}$ & $\begin{array}{l}\text { MEC- } \\
\text { Innov.1 }\end{array}$ & $\begin{array}{l}\text { Propuestas de diagnóstico de punto de venta (con } \\
\text { y sin subvención del gobierno regional) }\end{array}$ \\
\hline Fuente: Elaboración propia & \\
\hline
\end{tabular}

Una vez abreviado el elenco de propuestas de los PAC, se confeccionó una encuesta virtual para los veinticinco municipios elegidos de la provincia de Alicante. La encuesta fue enviada a mediados del año 2013 y el público objetivo fueron los técnicos AFIC (Agentes de Fomento de Iniciativas Comerciales) de los municipios que contaban con la redacción de un PAC.

En total participaron diez municipios: Calpe, San Vicente del Raspeig, Jávea, San Juan de Alicante, Elda, Almoradí, Denia, Novelda y las dos ciudades de Alicante y Elche, en las que además se desarrolló un trabajo mucho más detallado y minucioso. El cuadro 6 muestra los municipios participantes con el año de publicación de los PAC y sus actualizaciones. 


\begin{tabular}{|l|l|}
\hline $\begin{array}{l}\text { Cuadro 6. Municipios participantes en el estudio y año de publicación de los PAC } \\
\text { (actualizaciones) }\end{array}$ \\
\hline Alicante & 1998, actualizado en 2006 \\
\hline Almoradí & 2012 \\
\hline Calpe & $2007-2013$ \\
\hline Denia & 2009 \\
\hline Elche & $\begin{array}{l}\text { PATECO y 1er PAC FUTURELX.2001; } 2^{\circ} \text { PAC FUTU- } \\
\text { RELX 2009 }\end{array}$ \\
\hline Elda & 2002 \\
\hline Jávea & 2001 \\
\hline Novelda & 2010 \\
\hline San Juan de Alicante & 2011 \\
\hline San Vicente del Raspeig & 1998, y actualización 2004 \\
\hline FuentE: Elaboración propia & \\
\hline
\end{tabular}

La encuesta constaba de tres bloques de preguntas. El primero recogía 20 cuestiones y valoraba el grado de satisfacción de los técnicos comerciales municipales sobre las propuestas anteriormente presentadas (véanse los cuadros 1 al 5) a través de preguntas cerradas con respuestas organizadas según una escala Likert de 5 puntos desde 1 "no se han realizado estas propuestas en mi municipio" hasta 5 "muy satisfecho/a con estas propuestas". Además se incluía una última opción por si el entrevistado quería señalar algún aspecto concreto con respecto a la línea de actuación analizada. El segundo bloque incluía tres preguntas abiertas en las que se les solicitaba información precisa sobre el municipio y el año de elaboración del PAC. Por último, el último bloque estaba formado por 5 preguntas que valoraban la importancia y efectividad de las líneas de actuación de cada una de las propuestas presentadas en los cuadros 1 al 5 . En esta ocasión la escala utilizada fue de 10 puntos desde 1 "ninguna importancia o efectividad" hasta 10 "mucha importancia o efectividad".

La encuesta fue utilizada en las diez ciudades analizadas, aunque para el estudio pormenorizado de las ciudades de Alicante y Elche se reforzó la información con una serie de entrevistas en profundidad a técnicos municipales de ambas ciudades. Así, en estas ciudades, además de entrevistar a los técnicos AFIC, se realizaron un total de nueve entrevistas en profundidad a los principales actores económicos, asociaciones de comerciantes y responsables de la administración pública implicados en la elaboración o aplicación de los PAC. Concretamente las nueve entrevistas en profundidad se realizaron a las dos principales asociaciones de comerciantes de ambas ciudades y los propios técnicos de comercio locales, a los dos técnicos y al director de la Oficina PATECO, encargada de elaborar los PAC, y dependiente del Consejo Superior de Cámaras de Comercio, Industria 
y Navegación de la Comunidad Valenciana, a la jefa del Servicio Territorial de Comercio en Alicante y, finalmente, a una consultora especializada en temas comerciales a escala regional.

\section{II.1 Objetivo e hipótesis de trabajo}

El objetivo principal de esta investigación ha sido realizar un análisis exploratorio para conocer el impacto de los PAC en los municipios donde se han realizado dichos estudios. La marcación de este objetivo venía dada por varias hipótesis de trabajo planteadas en la fase inicial de la investigación y que se traducen en las siguientes cuestiones de investigación:

1. ¿Verdaderamente los PAC tienen capacidad, si no para ejecutar sus propias propuestas, al menos para motivar a los equipos de gobierno locales para que las ejecuten?

2. ¿Las propuestas han estado financiadas y apoyadas en su totalidad por algún organismo competente en materia comercial?

3. Y si no es así, ¿qué posibilidad hay de que las administraciones locales las emprendan?

4. ¿Las ejecuciones de las propuestas han sido similares en todos los municipios?

5. ¿La priorización de algunas propuestas frente a otras ha acabado creando escenarios urbano-comerciales muy distintos?

Para esta investigación se ha utilizado información primaria y secundaria. Las entrevistas en profundidad y las encuestas de opinión han sido las principales fuentes de información primaria utilizadas. En cuanto a la información secundaria, los PAC de todos los municipios analizados han sido de indiscutible ayuda para delimitar las líneas estratégicas de actuación de la Oficina PATECO en el territorio. Además, se han revisado los balances municipales de las acciones e inversiones guiadas por los PAC, que han sido decisivos para conocer todo el proceso interno de priorización de las propuestas, de ejecución y de valoración de los resultados. Finalmente el soporte teórico está reflejado en el apartado de referencias bibliográficas. 


\section{LOS PAC EN LA PROVINCIA DE ALICANTE: EL CASO DE ALI- CANTE Y ELCHE}

La provincia de Alicante acogió los dos primeros proyectos PAC de toda la Comunidad Valenciana en el año 1998. Las ciudades de Alicante e Ibi fueron los primeros laboratorios donde se testó la metodología de análisis que más tarde se ha repetido por gran parte del territorio valenciano (EsPINOSA, 2007). En los años posteriores, se siguió trabajando mucho en la provincia de Alicante, en ciudades como San Vicente del Raspeig, Elche, Calpe o Jávea, escogiéndose en los últimos años municipios de menor dimensión y capacidad de atracción comercial.

En el caso de la ciudad de Elche, el PAC de PATECO se alternó con el plan FUTURELX, publicado por primera vez el mismo año en el que se publicó el PATECO, en 2001, y actualizado de nuevo en el año 2009. La ciudad de Elche cogió como base el proyecto del PATECO para generar el plan estratégico local de la ciudad.

El sistema de ciudades alicantino, caracterizado por la presencia de dos ciudades de mayores dimensiones, Elche y Alicante, presenta un gran número de ciudades medias y pequeñas, que ha permitido la creación de una red comercial policéntrica jerarquizada en la que la capitalidad la ejerce la capital provincial.

A continuación se presentan los resultados obtenidos en esta investigación atendiendo a las hipótesis de trabajo planteadas.

\section{RESULTADOS DE LA INVESTIGACIÓN}

\section{IV.1 Capacidad de los PAC para proponer las acciones de mejora urbano- comercial propuestas.}

Como ya se ha comentado anteriormente, los PAC no deciden, ni vinculan ni ejecutan las acciones que proponen para mejorar el tejido urbano-comercial de las ciudades seleccionadas. Son los ayuntamientos los que deciden qué acciones van a desarrollar, cuándo y cómo. De hecho, desde el PAC sólo se plantea la idea, mientras que el gobierno local se encarga de materializarla con todos los detalles de calidades y estética que ello conlleva.

Por eso, en relación con la primera cuestión de investigación sobre si los PAC tienen capacidad para motivar a los equipos de gobierno locales a que las 
ejecuten, las opiniones de los AFICs entrevistados son algo dispares (véase el cuadro 7).

\begin{tabular}{|c|c|c|c|c|c|c|}
\hline \multicolumn{7}{|c|}{ Cuadro 7. Propuestas de urbanismo comercial (UC) } \\
\hline $\begin{array}{l}\text { Relacionada } \\
\text { con: }\end{array}$ & $\begin{array}{l}\text { Código de } \\
\text { propuesta }\end{array}$ & A & $\mathrm{B}$ & $\mathrm{C}$ & $\mathrm{D}$ & $\mathrm{E}$ \\
\hline \multirow{3}{*}{ Aparcamientos } & UC-Ap.1 & 4 & - & - & 4 & 1 \\
\hline & UC-Ap. 2 & 3 & 1 & 2 & 3 & 1 \\
\hline & UC-Ap. 3 & 7 & - & - & 2 & 1 \\
\hline \multirow{5}{*}{$\begin{array}{l}\text { Mobiliario ur- } \\
\text { bano }\end{array}$} & UC-Mob. 1 & 2 & - & 1 & 3 & 4 \\
\hline & UC-Mob. 2 & 2 & - & - & 3 & 4 \\
\hline & UC-Epúb. 1 & 3 & - & 1 & 3 & 1 \\
\hline & UC-Epúb. 2 & 5 & - & - & 3 & 2 \\
\hline & UC-Epúb. 3 & 3 & - & 1 & 2 & 4 \\
\hline \multirow{2}{*}{\multicolumn{7}{|c|}{$\begin{array}{l}\text { No se han realizado estas propuestas en mi municipio; (B) Nada satisfecho con esta } \\
\text { propuestas } \\
\text { (C) Poco satisfecho con estas propuestas; (D) Satisfecho con estas propuestas; (E) } \\
\text { Muy satisfecho con estas propuestas }\end{array}$}} \\
\hline & & & & & & \\
\hline \multicolumn{7}{|c|}{ FUENTE: Elaboración propia } \\
\hline
\end{tabular}

Las propuestas de urbanismo comercial están relacionadas con tres aspectos clave, las zonas de parking, el mobiliario urbano y el embellecimiento de los espacios públicos. En cuanto a las preguntas relacionadas con los aparcamientos se ha detectado cierta polaridad con la apertura de nuevos aparcamientos públicos. La mitad de la muestra indica que no se han abierto nuevos aparcamientos en sus municipios, mientras que el resto opina que sí están satisfechos o muy satisfechos con esta actuación. Además, los AFIC distinguían qué aparcamientos municipales se han abierto al margen de las recomendaciones del PAC, con financiación exclusivamente local. En la ciudad de Elche, la mayoría de los aparcamientos que se realizaron en la zona central de la ciudad se hicieron como recomendación del Plan Estratégico Futurelx y no siguiendo las recomendaciones del PAC, siendo además la financiación puramente municipal. También, en el caso de Alicante, uno de los aparcamientos vertebradores de la ciudad, el situado en la Avenida Alfonso X El Sabio, se planteó en el PAC, aunque finalmente no se dotó de financiación a la ciudad para su ejecución.

En cuanto a la colocación de bolardos o pilonas para evitar los aparcamientos, sólo uno de los entrevistados indica que no está satisfecho en absoluto con esta acción, mientras que dos de ellos lo están ligeramente. En los últimos años, muchos de los proyectos PAC han substituido las recomendaciones de pi- 
lonas o bolardos por otras soluciones menos costosas que separan las aceras de la calzada. De hecho, en la ciudad de Alicante se ha evitado colocar bolardos en las nuevas calles de coexistencia, intercalando jardineras o luminarias, que consiguen frenar el número de aparcamientos indebidos pero que tienen una doble función, de control del aparcamiento y de embellecimiento urbano. Además, como se recomendaba para esta misma ciudad, las primeras acciones del PAC debían incluir los bolardos para asegurar su éxito, porque en la ciudad había una tendencia muy acusada a ocupar cualquier espacio de acera que se pudiera. Con el aumento de las denuncias de infracciones, este comportamiento indebido se ha ido reduciendo paulatinamente.

Del sistema ORA, el 70\% de la muestra señala que no se han realizado estas propuestas en sus zonas. En los casos en los que sí se han realizado, dos de los entrevistados están satisfechos o incluso hay un municipio muy satisfecho. En Elche, los técnicos de comercio destacaban el malestar inicial de los comerciantes por la introducción de la ORA en las calles donde estaban sus establecimientos comerciales, aunque una vez se ponían en marcha estos sistemas de control ayudaban al ordenamiento del tráfico y a la rotación rápida de vehículos. De esta manera la sensación de una medida incómoda a priori pasaba a ser una medida bien aceptada.

En relación con el mobiliario urbano son dos las líneas de actuación que proponen los PAC. Por un lado la decoración y la señalización de edificios y zonas peatonales y, por otro, la iluminación. El $20 \%$ de la muestra señala que en sus municipios no han tenido constancia de ninguna actuación a este respecto mientras que el resto de AFICs están ciertamente satisfechos con las actuaciones acometidas. El cambio de luminarias antiguas por otras más eficientes y respetuosas con el entorno urbano correspondiente ha sido una de las actuaciones más repetidas, sobre todo en calles en las que la renovación ha sido total.

De hecho, en Alicante, las primeras obras de los años 1998 y 1999 estuvieron casi exclusivamente dedicadas a la iluminación, ya que se pedía más seguridad en las calles a este nivel y más eficacia de la iluminación.

Los espacios públicos se han abordado desde tres perspectivas: la ocupación de espacio por terrazas, la peatonalización y las calles de coexistencia. Llama la atención la peatonalización, medida que no se ha realizado en absoluto en la mitad de los municipios estudiados mientras que en los que sí se ha puesto en marcha, los resultados obtenidos son muy satisfactorios.

En los casos concretos de Alicante y Elche, los comerciantes preferían la reducción del tráfico rodado pero sin llegar a una plena peatonalización. El ejem- 
plo negativo de las calles Castaños y San Francisco en Alicante, peatonalizadas en los años noventa, y que han experimentado serios problemas económicos por la falta de accesibilidad y de espacios de estacionamiento cercanos, ha provocado una oposición casi absoluta a cualquier proyecto que contemple la peatonalización total de una calle.

Lo que sí se ha realizado ha sido la ampliación de aceras y la reducción del número de carriles de circulación rodada al mínimo, para no restar accesibilidad pero evitar un ruido y tráfico excesivo en calles no preparadas para ello, sobre todo en el centro urbano.

Referente a la línea de actuación sobre la optimización de la ocupación de espacio público por terrazas (UC-Epúbl1) uno de los AFIC valora literalmente que para los vecinos esta línea de actuación ha resultado fatal, no pronunciándose en ninguna de las respuestas predeterminadas, mientras que otro recalca que en los últimos años la ocupación de espacio público está llegando a ser abusiva, no respetando en algunos casos los horarios de cierre.

En Alicante, la proliferación de terrazas ha supuesto un revulsivo económico para el centro histórico, ya que muchos nuevos bares y restaurantes han substituido al antiguo comercio que poco a poco había desaparecido. Por ese motivo, en el ayuntamiento de esta ciudad son conscientes de que esta nueva situación ha supuesto una mejora substancial para los comerciantes, pero la otra cara de la moneda es que ha sido negativa para los vecinos.

Las dos propuestas de mejora relacionadas con la gestión comercial (véase el cuadro 8) reflejan que los responsables están satisfechos con las actuaciones realizadas en relación con el fomento del asociacionismo comercial (siete de los entrevistados), mientras que en cuanto a los cursos de formación, casi la mitad indica que no se han realizado en su municipio. Señalamos la respuesta de uno de los AFICs que en cuanto al grado de asociacionismo indica que se ha realizado a pesar de los esfuerzos, la excesiva atomización de las asociaciones por barrios, gestión del día a día más que enfoques a medio y largo plazo.

Concretamente, en la ciudad de Alicante, la Agencia de Desarrollo Local contaba con un departamento de formación que se encargaba de realizar, promover y gestionar los cursos de formación a comerciantes propuestos en el PAC. A pesar de que se mantuvieron durante muchos años, uno de los problemas principales era que la asistencia nunca fue masiva y por tanto, no hubo trasferencia de conocimientos a la comunidad de comerciantes. 
Algunos técnicos han sido críticos con las ayudas para la promoción del asociacionismo comercial, ya que durante un largo período de tiempo se subvencionó la creación de nuevas asociaciones, provocando la proliferación de asociaciones que incluso llegaron a superponerse espacialmente, y que tenían una actividad casi nula.

\begin{tabular}{|c|c|c|c|c|c|c|}
\hline \multicolumn{7}{|c|}{ Cuadro 8. Propuestas de mejora de la gestión comercial (GC) } \\
\hline $\begin{array}{l}\text { Relacionada } \\
\text { con: }\end{array}$ & $\begin{array}{l}\text { Código de } \\
\text { propuesta }\end{array}$ & A & B & $\mathrm{C}$ & $\mathrm{D}$ & $\mathrm{D}$ \\
\hline Formación & GC-Form 1 & 4 & & 3 & 3 & \\
\hline Asociacionismo & GC-Aso1 & 1 & - & 1 & 7 & - \\
\hline \multicolumn{7}{|c|}{$\begin{array}{l}\text { No se han realizado estas propuestas en mi municipio; (B) Nada satisfecho con estas } \\
\text { propuestas } \\
\text { (C) Poco satisfecho con estas propuestas; (D) Satisfecho con estas propuestas; (E) } \\
\text { Muy satisfecho con estas propuestas }\end{array}$} \\
\hline \multicolumn{7}{|c|}{ FUENTE: Elaboración propia } \\
\hline
\end{tabular}

Las propuestas de dinamización comercial se enfocan a nivel general (véase el cuadro 9) y a nivel turístico (véase el cuadro 10). Las respuestas relacionadas con la imagen de una marca comercial local y de rutas comerciales reflejan todo tipo de opiniones, estando los entrevistados inclinados por todo tipo de respuestas posibles. De forma específica uno de ellos no se pronuncia al respecto e indica para DC-Img1 Sin opinión. Se han realizado campañas de promoción de compras en la ciudad y atracción comarcal pero no con marca local propia. Otro de los entrevistados responde que lo referente a la creación de rutas comerciales peatonales (DC-Img2), están en ejecución actualmente.

En Alicante, en general, no se han desarrollado grandes acciones encaminadas a desarrollar la imagen de marca, salvo la creación en la web municipal de rutas comerciales urbanas (véase http://alicante-rutasurbanas.blogspot.com.es/) y otras acciones de pequeño calado.

Sin embargo, en la actualidad, y fuera del PAC, la llegada del AVE se ha interpretado como una oportunidad para promocionar la ciudad desde un punto de vista comercial y para incrementar también los flujos turísticos y comerciales. Así, desde el inicio de la conexión Madrid-Alicante se reparten guías comerciales en la propia estación del AVE en Madrid a los pasajeros.

En Elche, sin embargo, se puso en marcha un ambicioso proyecto de Centro Comercial Abierto (CCA) en el centro urbano de la ciudad. Con este CCA, se dinamizó la zona centro de forma integral y se consiguió mejorar la percepción 
de los usuarios de la misma a través de subvenciones a la principal asociación de comerciantes de la ciudad. En el año 2011 la asociación de comerciantes se vio forzada a paralizar el proyecto por la interrupción de las ayudas financieras que recibían.

Las opiniones sobre las actuaciones relacionadas con la comunicación realizada por el municipio reflejan que, en general sí se han llevado a cabo y que son especialmente las ferias y las ferias outlet (DC-Com2) las que más grado de satisfacción han encontrado entre los participantes, si tenemos en cuenta las opiniones favorables. La creación de guías comerciales (DC-Com3) ha sido una propuesta de dinamización comercial que ha contado también con mucha aceptación (4 AFICs muy satisfechos y 2 de ellos satisfechos).

\begin{tabular}{|c|c|c|c|c|c|c|}
\hline \multicolumn{7}{|c|}{ Cuadro 9. Propuestas de dinamización comercial (DC) } \\
\hline $\begin{array}{l}\text { Relacionada } \\
\text { con: }\end{array}$ & $\begin{array}{l}\text { Código de } \\
\text { propuesta }\end{array}$ & A & $\mathrm{B}$ & $\mathrm{C}$ & $\mathrm{D}$ & $\mathrm{E}$ \\
\hline \multirow{2}{*}{ Imagen } & DC-Img.1 & 2 & 1 & 2 & 2 & 2 \\
\hline & DC-Img. 2 & 3 & - & 1 & 3 & 2 \\
\hline \multirow{3}{*}{ Comunicación } & DC-Com.1 & 2 & - & 3 & 2 & 3 \\
\hline & DC-Com.2 & - & 1 & - & 5 & 4 \\
\hline & DC-Com.3 & 1 & - & 3 & 2 & 4 \\
\hline \multicolumn{7}{|c|}{$\begin{array}{l}\text { No se han realizado estas propuestas en mi municipio; (B) Nada satisfecho con estas } \\
\text { propuestas } \\
\text { (C) Poco satisfecho con estas propuestas; (D) Satisfecho con estas propuestas; (E) } \\
\text { Muy satisfecho con estas propuestas }\end{array}$} \\
\hline \multicolumn{7}{|c|}{ FUENTE: Elaboración propia } \\
\hline
\end{tabular}

Las propuestas más específicas relacionadas con la dinamización turística no se han realizado en tres de los diez municipios en ningún sentido (ni creación de rutas turístico comerciales (DTC-Img1), ni animaciones y promociones turístico comercial conjunta con hoteles y restaurantes (DTC-Com1). La falta de financiación para desarrollar acciones que tienen un coste económico bastante elevado ha frenado muchos de estos nuevos proyectos.

En cualquier caso, son estas últimas con las que más satisfechos están los AFICs de los municipios entrevistados (véase el cuadro 10). De forma precisa, uno de los entrevistados señala con respecto a DTC-Com1 "esfuerzos por vincular con el Parque Empresarial de Elche y En fase de r edacción actualmente". 


\begin{tabular}{|c|c|c|c|c|c|c|}
\hline \multicolumn{7}{|c|}{ Cuadro 10. Propuestas de dinamización turística comercial (DTC) } \\
\hline $\begin{array}{l}\text { Relacionada } \\
\text { con: }\end{array}$ & $\begin{array}{l}\text { Código de } \\
\text { propuesta }\end{array}$ & A & B & $\mathrm{C}$ & $\mathrm{D}$ & $\mathrm{E}$ \\
\hline Imagen & DTC-Img.1 & 3 & - & 2 & 3 & - \\
\hline Comunicación & DTC-Com.1 & 3 & - & 2 & 3 & 2 \\
\hline \multicolumn{7}{|c|}{$\begin{array}{l}\text { No se han realizado estas propuestas en mi municipio; (B) Nada satisfecho con esta } \\
\text { propuestas } \\
\text { (C) Poco satisfecho con estas propuestas; (D) Satisfecho con estas propuestas; (E) } \\
\text { Muy satisfecho con estas propuestas }\end{array}$} \\
\hline \multicolumn{7}{|c|}{ FUENTE: Elaboración propia } \\
\hline
\end{tabular}

Los planes recogen las propuestas de modernización de los establecimientos comerciales basándose principalmente en aspectos tecnológicos, de infraestructuras de los locales y de mejoras en los puntos de venta a través del diagnóstico del punto de venta. En relación a todas ellas, en general los entrevistados están poco satisfechos con las mismas, si bien es cierto que se han acometido prácticamente en la totalidad de municipios (véase el cuadro 11).

Hasta el año 2008, el gobierno regional subvencionó la introducción de las TICs a aquellos comercios que se acogieran a las ayudas programadas. Este proyecto funcionó bien hasta hace relativamente dos años, cuando ha cesado su funcionamiento por falta de presupuesto.

\begin{tabular}{|c|c|c|c|c|c|c|}
\hline \multicolumn{7}{|c|}{ Cuadro 11. Propuestas de modernización de establecimientos comerciales (MEC) } \\
\hline $\begin{array}{l}\text { Relacionada } \\
\text { con: }\end{array}$ & $\begin{array}{l}\text { Código de } \\
\text { propuesta }\end{array}$ & A & B & $\mathrm{C}$ & $\mathrm{D}$ & $\mathrm{E}$ \\
\hline Teconología & MEC-Tec. 1 & 2 & - & 3 & 3 & 1 \\
\hline Infraestructuras & MEC-Inf.1 & 1 & 1 & 4 & 4 & - \\
\hline $\begin{array}{l}\text { Retail innova- } \\
\text { tion }\end{array}$ & $\begin{array}{l}\text { MEC-In- } \\
\text { nov.1 }\end{array}$ & 1 & - & 3 & 3 & 1 \\
\hline \multicolumn{7}{|c|}{$\begin{array}{l}\text { No se han realizado estas propuestas en mi municipio; (B) Nada satisfecho con esta } \\
\text { propuestas } \\
\text { (C) Poco satisfecho con estas propuestas; (D) Satisfecho con estas propuestas; (E) } \\
\text { Muy satisfecho con estas propuestas }\end{array}$} \\
\hline \multicolumn{7}{|c|}{ FUENTE: Elaboración propia } \\
\hline
\end{tabular}

Finalmente, en el cuadro 12 se reflejan las valoraciones de los diez AFICs sobre la importancia y efectividad de las propuestas y acciones emprendidas por los PAC. La línea mejor valorada estadísticamente es la dinamización comercial con una media de 7,55 sobre 10 puntos y un mínimo de 3 , con una ligera desvia- 
ción típica de 1,87. A continuación la siguiente mejor valorada es el urbanismo comercial con un 6.62, aunque encuentra puntuaciones de 0 en su valoración. La variable menos valorada por los entrevistados ha sido la que hace referencia a la modernización de los establecimientos comerciales.

\begin{tabular}{|c|c|c|c|c|c|c|c|}
\hline $\begin{array}{l}\text { Cuadro 12. Val } \\
\text { nes }\end{array}$ & $a c i$ & nes sobre & importa & a y efe & idad & las propu & tas y accio- \\
\hline Variable & $\mathrm{N}$ & Mínimo & Máximo & Media & $\begin{array}{l}\text { Error } \\
\text { típico }\end{array}$ & Varianza & $\begin{array}{l}\text { Desvia- } \\
\text { ción típica }\end{array}$ \\
\hline $\begin{array}{l}\text { Mejora de la } \\
\text { gestión co- } \\
\text { mercial }\end{array}$ & 10 & .00 & 10.00 & 6,10 & .84 & 7.21 & 2.68 \\
\hline $\begin{array}{l}\text { Dinamización } \\
\text { comercial }\end{array}$ & 9 & 3.00 & 9.00 & 7,55 & 0.62 & 3.52 & 1.87 \\
\hline $\begin{array}{l}\text { Dinamización } \\
\text { turístico co- } \\
\text { mercial }\end{array}$ & 9 & 2.00 & 8.00 & 6.16 & 0.62 & 3.50 & 1.87 \\
\hline $\begin{array}{l}\text { Moderniza- } \\
\text { ción de esta- } \\
\text { blecimientos } \\
\text { comerciales }\end{array}$ & 10 & .00 & 9.00 & 5.50 & 0.95 & 9.16 & 3.02 \\
\hline $\begin{array}{l}\text { Urbanismo } \\
\text { comercial }\end{array}$ & 8 & .00 & 9.00 & 6.62 & 1.05 & 8.83 & 2.97 \\
\hline
\end{tabular}

\section{IV.2 Financiación de los PAC y financiación de las obras y propuestas}

La financiación es un aspecto clave en la ejecución de las acciones propuestas en los Planes de Acción Comercial. De hecho, los técnicos del PATECO afirmaron que los ayuntamientos valencianos solicitaban la redacción de los PAC a la Consellera por tres motivos fundamentales:

1. para conseguir estudios estratégicos

2. para conseguir financiación en el pago del propio proyecto PAC y

3. para tener una posición privilegiada a la hora de conseguir subvenciones que sufragaran obras locales que, de otro modo, habrían sido imposibles de realizar con fondos públicos locales.

De hecho, hasta la puesta en marcha de los PAC, la Generalitat Valenciana, a través de la Conselleria de Economía, Industria, Turismo y Empleo financiaba los proyectos que solicitaban los ayuntamientos que así consideraran oportuno, 
a consultoras privadas. La entrada de los PAC supuso la reducción de los gastos en investigación comercial por empresas externas a la administración pública y sobre todo, la utilización de una misma metodología de actuación para todos los municipios (realización de encuestas y entrevistas, creación de comisiones mixtas y de seguimiento o estudio de las mismas variables en todos los municipios).

El primer PAC que realizó la Oficina PATECO, el PAC Lucentum, en la ciudad de Alicante fue sufragado íntegramente por la Generalitat Valenciana, pero tras esta primera experiencia piloto, los $\mathrm{PAC}$ han sido cofinanciados entre el Ayuntamiento o mancomunidad (en el caso de los PAC que tienen una escala supramunicipal) y la Generalitat Valenciana. En la actualidad, ésta última está llegando a financiar entre el 50 y $70 \%$ del coste total del proyecto, que en cualquier caso no rebasaría los 30.000 euros/año. La Oficina PATECO recomienda a los ayuntamientos realizar el PAC un lustro antes de dar comienzos con las obras: Depende de su grado de ejecución y de la propia evolución del tejido comercial local. Nuestra experiencia indica que es conveniente realizar una actualización a partir de los 5-7 años de su r ealización anterior (www.pateco.org). Evidentemente, los gastos del proyecto eran menores cuando se utilizaban los recursos de los que ya disponía la administración local.

Una vez realizado y publicado el PAC, los ayuntamientos que solicitasen a la Conselleria de Economía, Industria, Turismo y Empleo una subvención para llevar a cabo cualquier propuesta que les indicaba el PAC, tenían prioridad frente a aquellos municipios que planteaban acciones por su cuenta o, en el caso de que contaran con un PAC, que planteasen ejecutar propuestas que no habían sido programadas ni recogidas en los PAC.

Así, las actuaciones derivadas de la ejecución del Plan de Acción Comercial cuentan con el apoyo técnico y financiero de la Generalitat en actuaciones de promoción comercial, urbanismo, mejora de mercados, etc" (www.pateco.org).

En el caso del urbanismo comercial, y debido al coste de las partidas presupuestarias, había una vinculación directa entre la concesión de las subvenciones y la existencia de un PAC.

Para otras propuestas, muchos ayuntamientos han combinado estas subvenciones regionales con la captación de fondos desde otros organismos o programas, tales como el Plan E, el Plan Confianza o las inversiones locales a crédito.

Sin embargo, en los últimos años, ha habido una reducción del volumen de subvenciones y de las partidas de las mismas, por lo que muchos ayuntamientos están a la espera de poder ejecutar sus acciones. Así, uno de los entrevistados 
señala que su PAC es muy reciente (2010) y que el Plan es extraordinario, pero durante estos últimos 4 años desde el Ayuntamiento de la ciudad no hemos podido desarrollar las medidas de urbanismo comercial que plantea por falta de consignación presupuestaria y por inexistencia de apoyo público en base a ayudas por parte de Conselleria".

Analizando de forma más detallada la financiación que la Generalitat Valenciana brindó a las ciudades de Alicante y Elche, es posible detectar una gran diferencia en los fondos asignados a cada ciudad, teniendo en cuenta que han ido disminuyendo conforme pasaban los años en ambos municipios.

Concretamente, Alicante recibió un total de 5.980.683,50€, sobre todo los primeros cinco años tras la publicación del PAC (véase la figura 1), que se centraron en la reurbanización de calles con vocación comercial, el soterramiento de contenedores de basura, acciones puntuales de urbanismo comercial por parte de la ciudad, centro y barrios periféricos, inversiones en iluminación y la reforma del Mercado Central de Alicante.

Figura 1. Financiación autonómica para la ciudad de Alicante

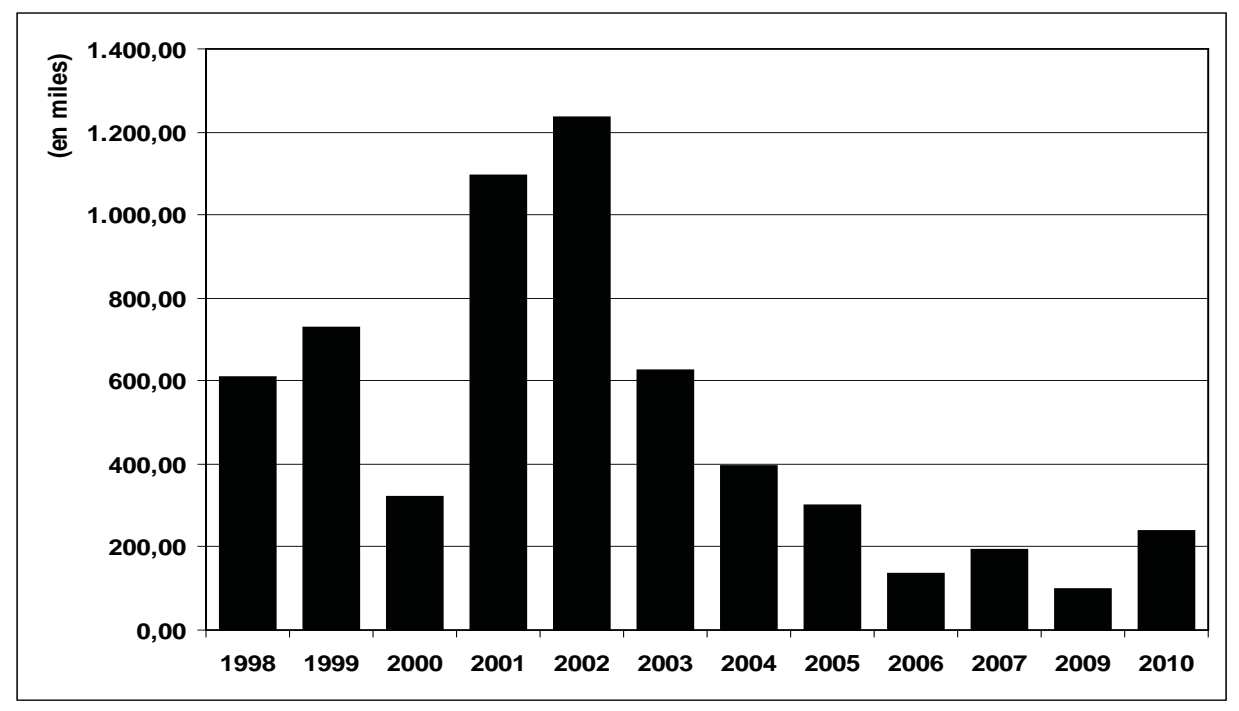

FuENTE: Servicio territorial de Comercio y Consumo en Alicante.

Debido a que la ciudad había acogido al primer proyecto piloto de la Comunidad Valenciana, hubo más predisposición para financiar y ejecutar las ac- 
ciones propuestas. Además, el PAC Lucentum fue un documento muy completo, pero que tampoco presentó un número excesivo de propuestas.

En el caso de Elche, la financiación autonómica fue mucho más modesta: 1.370.801,10€, conseguida sólo en cuatro anualidades (2001, 2002, 2005 y 2008) que se centraron en acciones de urbanismo comercial y en la peatonalización de una calle localizada en el centro urbano.

Las aportaciones económicas de las dos primeras anualidades fueron mucho mayores que las de los años 2005 y 2008, ya que el PAC acababa de publicarse y era necesario ejecutar obras de mayor envergadura, mientras que las aportaciones que se realizaron posteriormente fueron puntuales y como complemento a la partida presupuestaria que ya tenía planteada el Ayuntamiento de Elche para mejorar el espacio urbano.

Desde finales de la década de 1990, el Ayuntamiento de Elche ya había destinado partidas presupuestarias para fines comerciales, sin contar con las acciones futuras del PAC del PATECO y de la Generalitat Valenciana. Además, al seguir mayoritariamente el Plan de Acción Comercial realizado por la sociedad mercantil local Pimesa, dentro del plan estratégico Futurelx, muchas de las propuestas de las que solicitaron subvenciones no estaban vinculadas directamente al PAC realizado por la Oficina PATECO.

\section{IV.3 Ejecución de las propuestas en los municipios: ¿Similitud o disparidad?}

Respecto a si las ejecuciones de las propuestas han sido similares en todos los municipios hemos podido comprobar que, por el contrario, en muchas ocasiones han sido muy dispares. De hecho en algunos de ellos, no se han acometido en absoluto, mientras que en otros casos se han realizado con mucho éxito percibido por parte de los responsables.

Las acciones que no se han realizado en absoluto en la mayoría de los municipios son las que hacen referencia a las propuestas de urbanismo comercial. Curiosamente los AFICs señalan que las propuestas de modernización de establecimientos comerciales son las que, en general, se han realizado en mayor medida, pero con las que más descontentos están. Las razones principales habría que buscarlas en el menor valor de las partidas presupuestarias de estas acciones y su efectividad a muy corto plazo.

Las actuaciones sobre el mobiliario y la realización de eventos de venta en la calle como ferias, ferias outlet, etc... son aquellas con las que más satisfechos 
se sienten. Sobre todo, porque los comerciantes estaban satisfechos con este tipo de eventos multitudinarios que atraen público fuera de la rutina diaria comercial.

En cualquier caso, y en enlazando el punto anterior con éste, al priorizar determinadas acciones frente a otras, secuenciando su ejecución, se han creado escenarios urbano-comerciales dispares que atienden a multitud de elementos.

Así, una de las consecuencias más claras ha sido la creación de dos modelos de actuación siguiendo un esquema de dispersión y otro de concentración de acciones en el territorio.

Por ejemplo, San Vicente del Raspeig es, en opinión de la propia Oficina PATECO, uno de los mejores ejemplos en la ejecución casi absoluta de las propuestas del PAC en su centro urbano. Al concentrar todas las acciones del PAC en la zona central, la percepción de los ciudadanos de la renovación ha sido muy alta y a corto plazo. Es cierto que esta decisión de concentrar todas las acciones en un espacio reducido de la ciudad ha supuesto un proceso largo de obras en las principales calles comerciales de la ciudad, pero tras superarlo, han visto como la ciudad ha mejorado significativamente.

En el caso de la ciudad de Elche, tanto el PAC de PATECO como el realizado dentro de Futurelx abogaron por renovar el centro urbano comercial, evitando la dispersión de acciones por otras áreas urbanas. Aunque se han llevado acciones de reurbanización en calles de la zona del Ensanche, en el caso de Elche ha sucedido lo mismo que en San Vicente del Raspeig.

Alicante, sin embargo, decidió distribuir las propuestas por la zona centro y también los barrios, por lo que el efecto de renovación urbano-comercial se diluyó y no fue tan visible como en los dos casos anteriores.

\section{IV.4 La toma de decisiones municipal: priorización de las propuestas}

Una vez los PAC estaban finalizados, era ya potestad de los consistorios locales la decisión de qué hacer, cómo y cuándo hacerlo. Está claro que las acciones propuestas por los PAC tenían una gran relevancia en el embellecimiento de la ciudad, en la ganancia de accesibilidad, en la mejora de los principales ejes comerciales y en la renovación de áreas comerciales centrales en crisis. Acciones todas ellas que pueden otorgar directamente un rédito político a las corporaciones locales que estuvieran gobernando en el período de ejecución de los PAC. 
Por otra parte, son los ayuntamientos quienes mejor conocen sus necesidades a corto, medio y largo plazo y quienes deben gestionar el calendario de trabajo y ejecución de las obras previstas.

Por ese motivo, ni en un primer momento la Oficina PATECO indicaba qué obras eran más urgentes de acometer, ni una vez se solicitaban las subvenciones para realizar las obras, la Conselleria de Economía, Industria, Turismo y Empleo discutía la priorización que había organizado la administración local.

\section{CONCLUSIONES}

Los Planes de Acción Comercial pueden considerarse los primeros estudios que integran la actividad comercial dentro del territorio en la Comunidad Valenciana. El uso de una misma metodología de análisis y estudio ha simplificado su realización, permitiendo a técnicos e investigadores de la actividad comercial comparar municipios con realidades demográficas, sociales y económicas muy dispares.

Una de las primeras consecuencias directas de la elaboración de los PAC, ha sido la de dotar a los municipios de monografías locales con datos actualizados y elaborados teniendo en cuenta una gran cantidad de variables.

Sin embargo, a pesar del gran potencial que supone un PAC para un municipio la ejecución de las propuestas (casi siempre de cierta envergadura económica y con diferentes agentes implicados) están limitadas a las posibles subvenciones que pueda beneficiarse el municipio y a las decisiones que priorice el equipo de gobierno del ayuntamiento en cuestión.

Lo más ventajoso es que estamos ante unos documentos de trabajo de especial valor para los municipios, radiografías que analizan su realidad para mejorarla y mejorar la vida de sus ciudadanos. Además, como se trata de documentos e investigaciones realizadas con una metodología común en su elaboración, se pueden aprovechar las economías de escala que ello supone entre municipios.

Finalmente, la priorización de algunas propuestas frente a otras ha acabado creando escenarios urbano-comerciales muy distintos en la geografía urbana de la provincia de Alicante. En ocasiones muy positivos, pero en otras no tanto. El toque de atención es que las ideas, la forma para ejecutarlas, y las iniciativas están sobre el papel, sobre unos documentos rigurosos que podrían ser aplicables a todo el territorio con la mejora a todos los niveles que para todos nosotros supondría. 
Los PAC aprueban el examen de evaluación de su razón de ser, pero para alcanzar la mejor calificación necesitan de un impulso por parte de todos los sectores.

Este trabajo no adolece de limitaciones ya que la muestra no recoge la totalidad de municipios, futuros trabajos podrían ampliar esta línea de investigación para conocer la realidad de todo el territorio y profundizar en aspectos más detallados de las acciones emprendidas.

\section{BIBLIOGRAFÍA}

Aldeiturriaga, J. (2004) "Comercio y usos terciarios en los centros tradicionales", ponencia presentada a las I Jornadas Ciudad y Revitalización de Centros Tradicionales, en Alicante, Diciembre.

Aparicio De Castro, María Gloria; Tejada Barrenetxea, Susana y Zorrilla Calvo, Pilar, "Dinamización del comercio urbano en España", Distribución y consumo, núm. 20, $\mathrm{N}^{\circ} 111,2010,40-51$. http://www.mercasa.es/files/multimedios/ pag_040-051_Aparicio-Tej_Zor.pdf

Espinosa Seguí, A. (2007): El comercio como herramienta de estructuración del territorio. El caso de las ciudades alicantinas. Tesis doctoral publicada en http://rua. ua.es/dspace/handle/10045/11453

Estevan, F.; Forés, D. y Hernández, C. (2007): GCU: Gestión de centros urbanos, Consejo de Cámaras Oficiales de Comercio, Industria y Navegación de la Comunidad Valenciana, 143.

Getz, D. (1993) “Tourist shopping villages: Development and planning strategies. Tourism Management, Vol.14, Num. 1, Febrero, pp 15-26.

Ley 3/2011, de 23 de marzo, de Comercio de la Comunidad Valenciana.

Murphy, L.; Benckendorff, P.; Moscardo,P. y Pearce, P.l. (2011) Tourist Shopping Villages: Forms and Functions. Routledge, London, NY, USA

Pérez, J.M. (1998). Urbanismo comercial y libertad de empresa, Ed. Marcial Pons, Ediciones Jurídicas y Sociales. Madrid.

Perles Ribes, José Francisco. "La regeneración urbana en la restructuración de destinos turísticos maduros: planificacion estratégica y gestión del conflicto. El caso de Calpe". En: Seminario Internacional Renovación y Reestructuración de Destinos Turísticos Consolidados del Litoral [Recurso electrónico]: comunicaciones / J. Fernando Vera Rebollo (ed.). Alicante: Universidad de Alicante, Instituto Universitario de Investigaciones Turísticas, 2011.

Planes de Accion Comercial de Alicante, Elche, Calpe, Denia, Jávea, Elda, San Joan D’alacant, San Vicente del Raspeig y Almoradí. Consejo de Cámaras de la Comunidad Valencia, Oficina Comercio y Territorio, Generalitat Valenciana, Conselleria d'Economia, Industria y Comercio (Disponibles en www.pateco. org). 
Rovira, A. (2000): "Propuesta y modelos para la gestión estratégica de los centros urbanos desde la cooperación pública y privada" en GAAP, n 19, 19-34.

Rovira, A.; Forés, D. y Hernández, C. (2012): Gestión innovadora de centros comerciales urbanos. Modelos y experiencias, ediciones Trea, Gijón, 284.

SCHILler, R. (1994): "Vitality and viability: challenge to the town centre", en International Journal of Retail and Distribution Management, vol. 22, nº, 46-50.

TARragó, M. (2012): “Urbanismo Comercial: Nuevas perspectivas en España y en Europa”, en BACHILLER MARTÍNEZ, Jesús, FRECHOSO REMIRO, Juan Carlos y VILLAREJO GALENDE, Helena (eds.): Jornadas Internacionales sobre urbanismo y gestión comercial, Universidad de Valladolid, Soria, págs. 1-12. http://www.uva.es/export/sites/default/contenidos/departamentos/economiaAplicada/documentos/JIUGC.pdf 